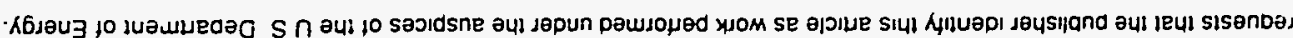

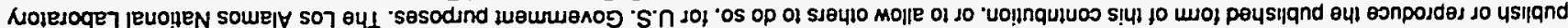

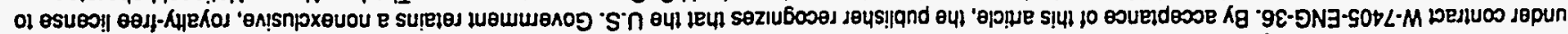

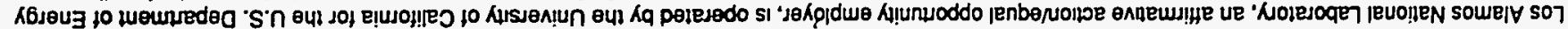

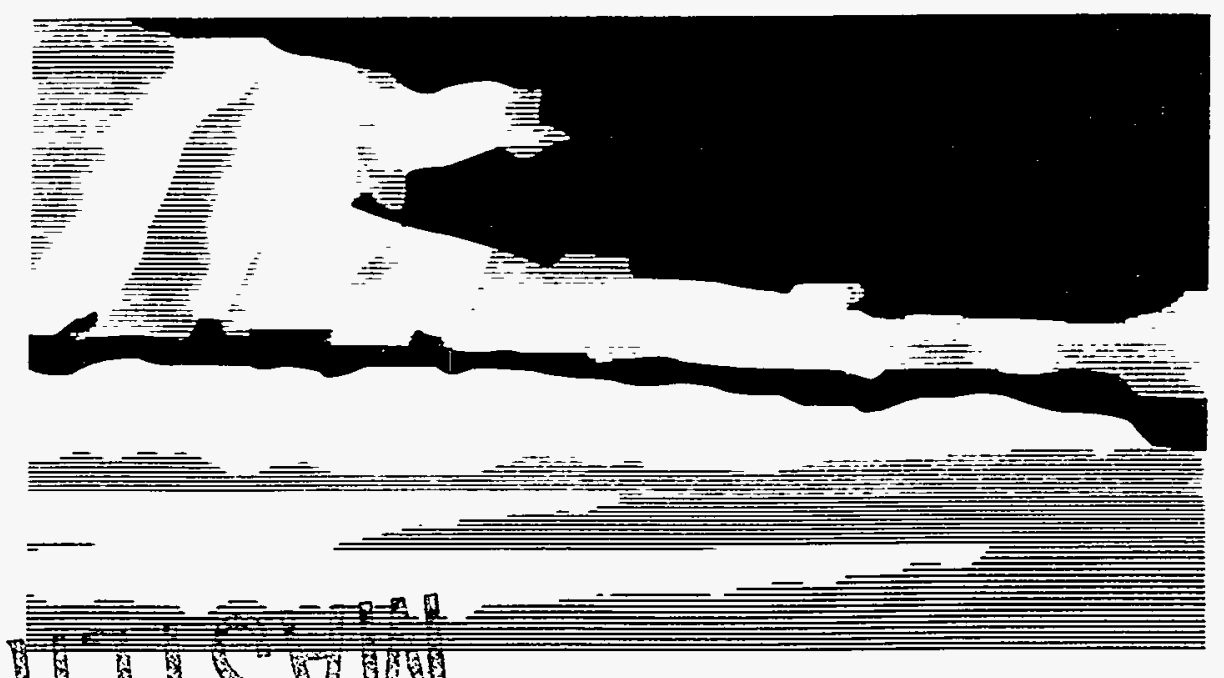

WN ‘

S66I ' $E T-0 T K T \mathrm{n}_{\Gamma}$

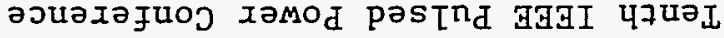

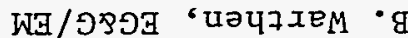

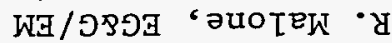

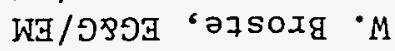

$$
\begin{aligned}
& \text { ođXd 'uosiapuy 'M } \\
& \text { WHX 'oगəțdeutues } \cdot \mathrm{V} \\
& \mathrm{HdX} \text { 'әә } \mathrm{T} \cdot \mathrm{H} \\
& \text { BdX 'sIamog } ~ T \cdot \mathrm{d} \\
& \text { हZ-d ' HozTEM • } \mathrm{d} \\
& \varepsilon Z-\mathrm{d} \text { 'uosuaxos } \alpha
\end{aligned}
$$

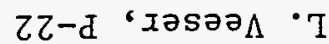

$$
\begin{aligned}
& \text { zz-d ' } \\
& \text { zz-d 's7feid } \cdot d \\
& \text { odd 'xayjed } \Lambda \cdot r
\end{aligned}
$$

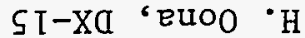

$$
\begin{aligned}
& \text { 乙Z-d 'usțpet - } 5 \\
& z z-\mathrm{d} \text { 'วuexuoo } r \\
& \eta z-d \text { ' yวszxeg 'd }
\end{aligned}
$$

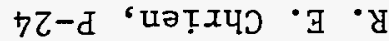

$$
\begin{aligned}
& \text { 乙て-d 'Кереяวон 'd ' }
\end{aligned}
$$

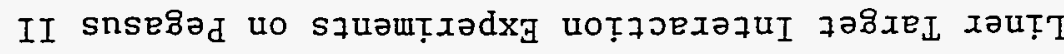

:ol pan!wans

:(s) Jout? $\forall$

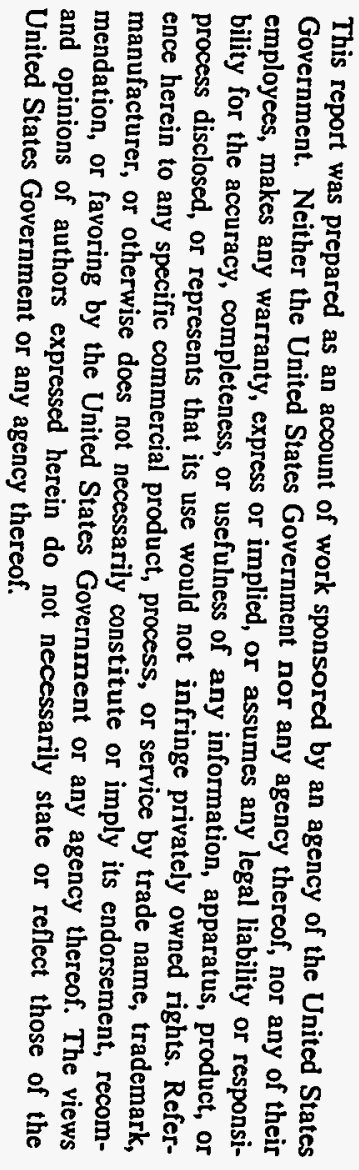

$:$ :|!!!

\section{0}

563662 네

$\prod_{i=0}=$ 


\section{DISCLAIMER}

Portions of this document may be illegible in electronic image products. Images are produced from the best available original document. 


\title{
LINER TARGET INTERACTION EXPERIMENTS ON PEGASUS II
}

\author{
M. P. Hockaday, R. E. Chrien, R. Bartsch, J. Cochrane, J. Ladish, H. Oona, J. V. Parker, D. Platts, J. Stokes, L. \\ Veeser, D. Sorenson, R. Walton, R. L. Bowers, H. Lee, A. Scannapieco, W. Anderson \\ Los Alamos National Laboratory \\ W. Broste, R. Malone, B. Warthen \\ EG\&G/EM, USA
}

The Los Alamos High Energy Density Physics program uses capacitively driven low voltage, inductive-storage pulse power to implode cylindrical targets for hydrodynamics experiments. Once a precision driver liner was characterized an experimental series characterizing the aluminum target dynamics was performed. The target was developed for shock-induced quasi-particle ejecta experiments including holography. The concept for the Liner shock experiment is that the driver liner is used to impact the target liner which then accelerates toward a collimator with a slit in it. A shock wave is set up in the target liner and as the shock emerges from the back side of the target liner, ejecta are generated. By taking a laser hologram the particle distribution of the ejecta are hoped to be determined. The goal for the second experimental series was to characterize the target dynamics and not to measure and generate the ejecta. Only the results from the third shot, Pegasus II-26 fired April 26th, 1994, from the series will be discussed in detail. The second experimental series successfully characterized the target dynamics necessary to move forward towards our planned quasi-ejecta experiments.

\section{Introduction}

The High Energy Density Physics Program at Los Alamos uses low voltage, inductive-storage pulsed power to implode cylindrical targets. This includes the $4.3 \mathrm{MJ}$ capacitor bank facility, Pegasus II. Several series of experiments have been performed to develop a liner driver and target ineteractions to investigate a variety of hydrodynamic physics issues such as assymetric flow, quasi-particle ejecta formation, and fluid instabilities. The initial series was to develop the driver that would allow us to reach shock pressures of $300 \mathrm{kbar}$. Solid cylindrical liners have been studied in other configurations and on other machines. ${ }^{1,2}$ The second liner series was to characterize the dynamics of a target liner, suitable for convergent ejecta experiments, as it is impacted by the driver liner.

The experimental goals of the first series were to uniformly accelerate a solid liner to $3 \mathrm{~mm} / \mathrm{ms}$ and to diagnose its time dependent shape, velocity, electrode to liner interface, and density. The $3 \mathrm{~mm} / \mathrm{ms}$ velocity is required to produce a nominally $300 \mathrm{kbar}$ shock in an aluminum target. The first series consisted of three shots: Liner 0 (Pegasus II-08) on April 5, 1993, Liner 1 (Pegasus II-09) on April 15, 1993, and Liner 2 (Pegasus II-10) on May 7 , 1993.

\section{Liner Experimental Configuration}

Each liner load was a $0.4 \mathrm{~mm}$ thick, $2 \mathrm{~cm}$ high, $4.8 \mathrm{~cm}$ diameter, 1100 series aluminum cylinder. Copper electrodes known as glide planes were cut at an angle of 8 degrees to keep the liner in contact as it imploded. Details of the fabrication of this load can be found in the companion paper, 17-7 by W. Anderson, et al. A $2.8 \mathrm{~cm}$ diameter, $2 \mathrm{~mm}$ thick, aluminum diagnostic pin cylinder was concentric with the liner and on the machine axis. The diameter of the pin cylinder was chosen to be just smaller than that of the calculated target. The liner configuration is shown in Figure 1.

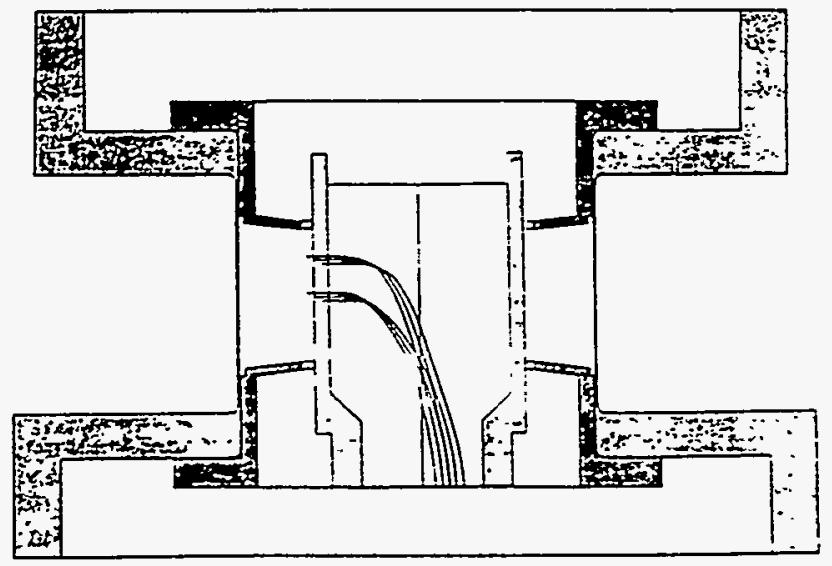

Figure 1. First Liner series load configuration showing the liner, copper electrodes, and pin cylinder.

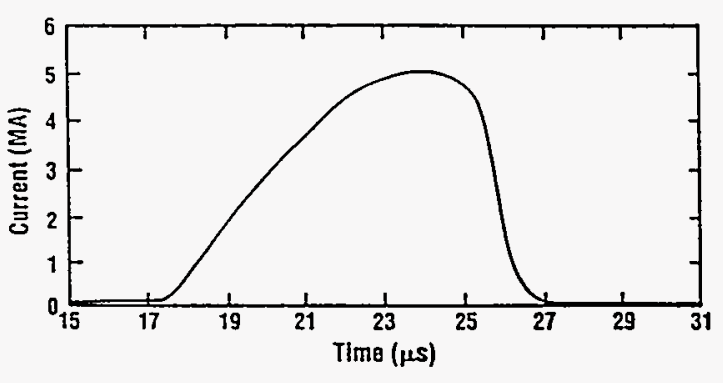

Figure 2. Current data from the top Rogowski Loop. The other diagnostics timed to start of current flow. 
The machine performance was diagnosed with Faraday rotation fibers. ${ }^{3}$ a Rowgowski loop, and B-dot probes. The machine was fired at $39 \mathrm{kV}$ with $5 \mathrm{MA}$ delivered to the load. A typical measured drive current from the Rogowski loop is shown in Figure 2.

The diagnostics used to observe the liner's dynamics were 24 optical pins, an optical framing camera, a high resolution gated imaging system, and triple axis radiography. A flux monitor measured the amount of magnetic flux that diffused through the liner as a function of time and was used to infer electrode integrity. The diagnostic port layout is shown in Figure 3.

The fiber optic closure pins were similar to the pins developed for high explosive experiments. ${ }^{4}$ Both bare fibers and fibers coupled to argon filled microballoons were tested. The fiber pins were used to measure the time of arrival of the liner at the fiber location, the velocity of the liner, and the symmetry of the liner implosion. Three configurations of fiber pins were used: fibers protruding $1 \mathrm{~mm}$ beyond the cylinder wall, fibers flush with the outer wall, and fibers in blind holes ending half way through the $2 \mathrm{~mm}$ thick wall. The light signal produced as the liner impacted the tip of the fiber was detected by photomultipliers with extremely non-linear bases to achieve a compressed gain and roughly five decades of dynamic range from below $1 \mu \mathrm{W}$ to above $10 \mathrm{~mW}$. A typical signal from a protruding pin is given in Figure 4.

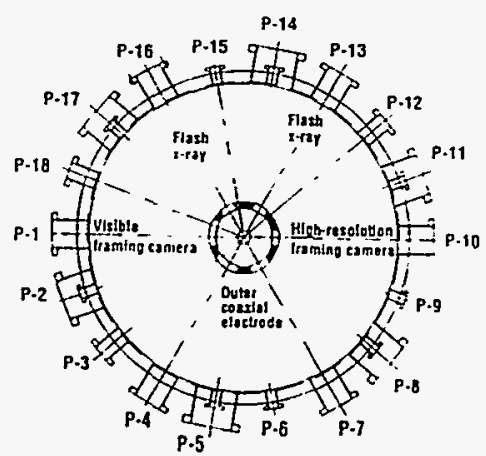

Figure 3. Diagnostic port layout. Also shown is the diagnostic window pattern in the initial outer coaxial electrode.

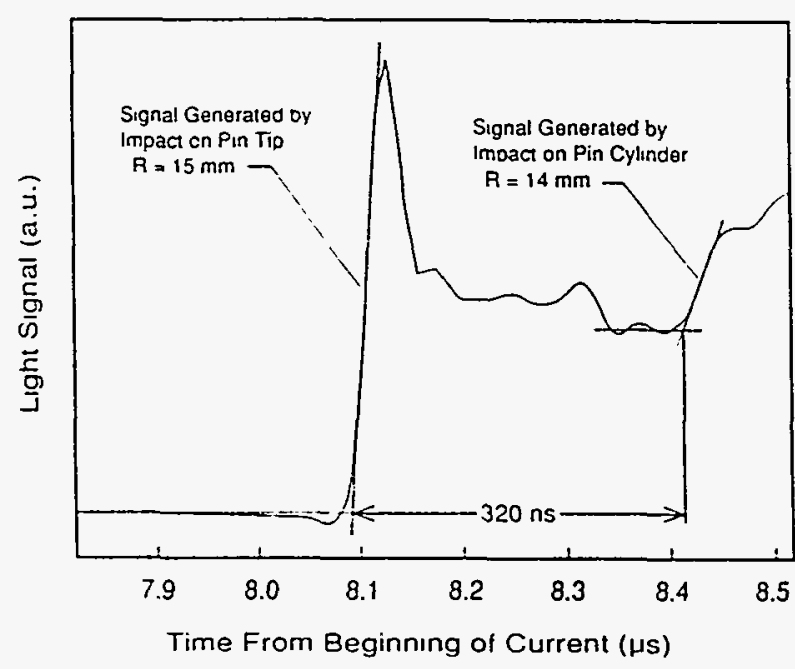

Figure 4. Signal recorded on protruding fiber pin at radius $15 \mathrm{~mm}$.

An Imacon 790 optical framing camera was used to observe the liner uniformity as a function of time. ${ }^{4}$ Twenty $100 \mathrm{~ns}$ frames were taken $500 \mathrm{~ns}$ apart. The objective of the system was to obtain two $90 \mathrm{~ns}$ gated high resolution images of the liner just before impacting the fiber pins. The resolution of this imaging system was $370 \mu \mathrm{m}$.

One axial and two radial flash $\mathrm{x}$-ray units were fielded to investigate the liner electrode interface, the liner uniformity, and the material state of the liner as it imploded. A schematic of the configuration is given in Figure 5. The $\mathrm{x}$-ray units employed a 12 stage, 12 Joule, $360 \mathrm{kV}$ Marx bank and could be independently triggered. ${ }^{5}$ The $\mathrm{x}$-ray units were modeled after similar units used on underground tests as backlighter and calibration sources. A $20 \mathrm{~ns}$ wide pulse of $5-30 \mathrm{keV} x$-rays was produced by identical windowless radial units coupled to the target chamber vacuum. Achieving acceptable contrast in the radial radiograph requires the low energy $x$-ray spectrum. The radial radiographs were recorded on $\mathrm{CX}$ and $\mathrm{DEF} \mathrm{x}$-ray film. The axial $\mathrm{x}$-ray unit utilized a commercial $\mathrm{x}$-ray tube with a carbon fiber cathode to emit a higher spectral energy since the $\mathrm{x}$-rays had to penetrate protective windows plus the two $1 \mathrm{~mm}$ copper glide planes. The axial radiographs were recorded CX and DEF film with a back scintillating screen of LANEX inside the vacuum chamber and XAR film with both a front and back QUANTA III scintillating screen outside the vacuum chamber. 


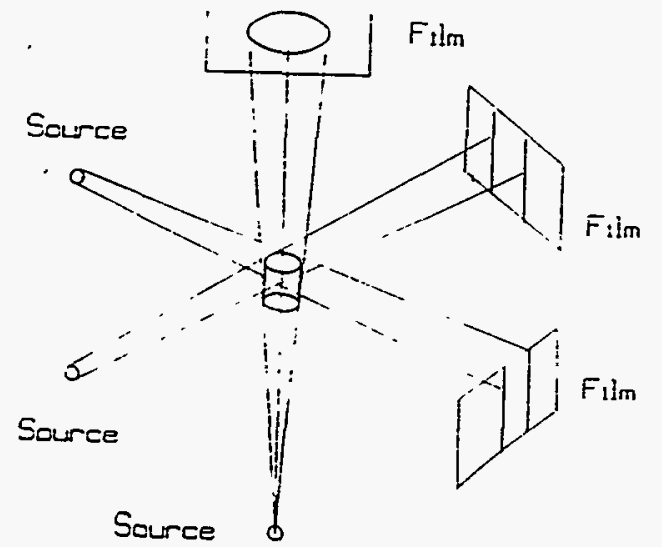

Figure 5. Triple-axis radiography schematic. The two radial views were 80 degrees apart.

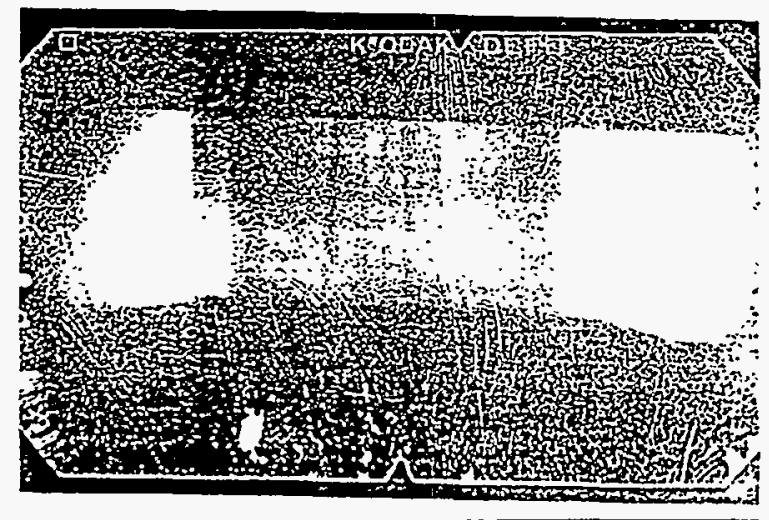

Figure 6.7.711 $\mu$ s radial view from Liner 1. The flux monitor wire, the pin cylinder edges, and the non-concentric liner are clearly visible.

\section{Liner 0}

Liner 0 was intended to determine if we could launch the solid liner and to check out diagnostics that were being developed specifically for this series. The optical pin data indicated that the liner was accelerated to $3.2 \mathrm{~mm} / \mu \mathrm{s}$, that the liner had a taper from top to bottom, and that there may have been $-0.1 \mathrm{~mm}$ irregularities in the inner surface of the liner on a $2 \mathrm{~mm}$ scale length. The optical framing camera data also indicated that there was a tapering of the liner. The two radial $\mathrm{x}$-ray units had been mistimed and data was obtained after the liner had impacted the pin cylinder.

\section{Liner 1}

Liner 1 was a repeat of Liner 0 as far as machine parameters were concerned. The optical pin configuration was optimized to investigate the possibility of the small scale perturbations on the liner inner surface and only bare fibers were used since the fibers with microballoons were harder to position and fabricate. The axial x-ray unit was fielded for the first time.

All the diagnostics returned good data. The optical and radial $x$-ray data indicated that the liner came in straight without a taper. The optical pin data and the $\mathrm{x}$-ray data showed that the liner and the pin cylinder were not concentric and that there was a small bulge in the liner. Figure 6 shows the radial view taken at $7.711 \mu \mathrm{s}$ after start of current flow and clearly shows the asymmetry of the implosion. A blowup of the radial view is shown in Figure 7 detailing the liner-electrode interface and the corresponding prediction made with our two-dimensional eulerian code is given in Figure 8.

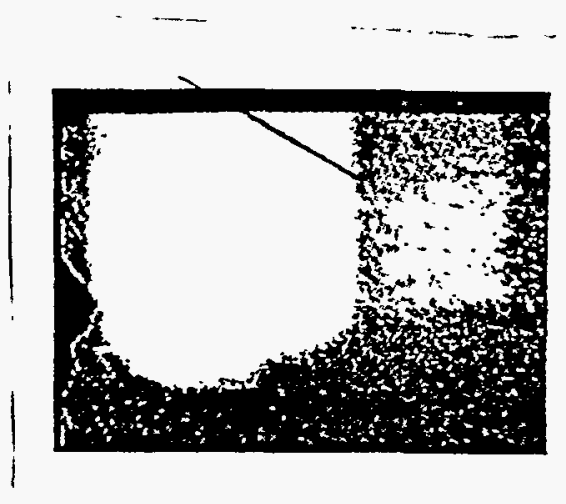

Figure 7. Blowup of Figure 6 showing details of the liner electrode-interface.

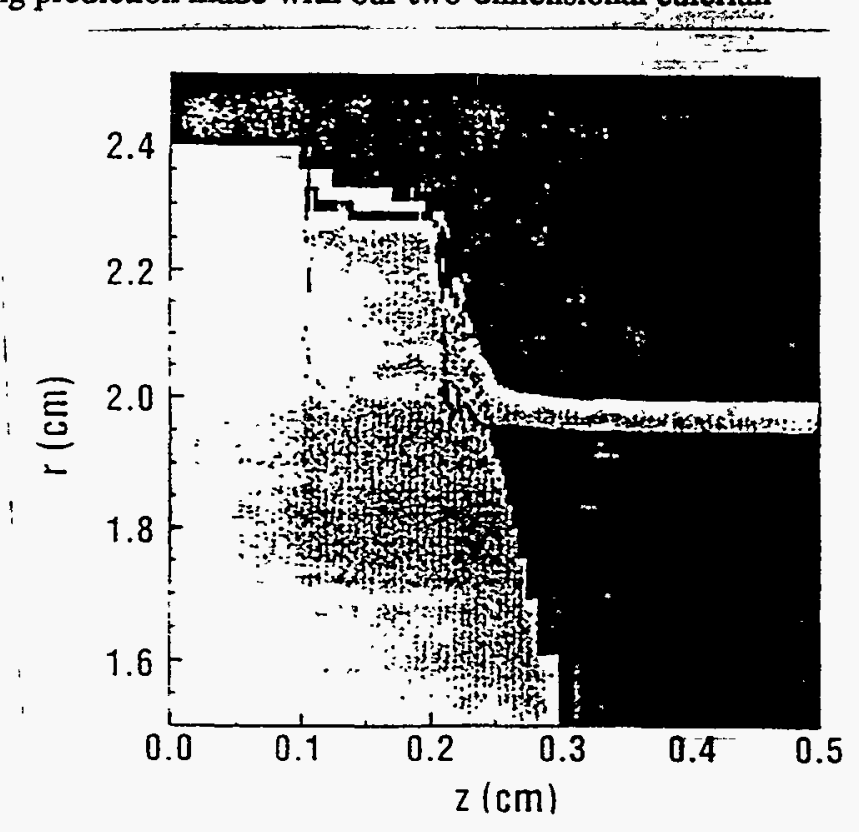

Figure 8. Two-dimensional simulation of the linerelectrode contact. 
Liner 2

It was hypothesized that the assymetry of the diagnostic windows could have caused the asymmetry in the liner implosion. $A$ bench experiment was set-up 10 measure the $B^{2}$ field asymmetry produced by the diagnostic windows through the retum current path. A ? rield asymmetry was found. Calculations showed that this magnitude could accoun for the observed implosion ' L'e. 1993). The diagnostic window configuration was changed to have six-fold symmetry as shown in Figure $y$ for Liner 2. For Liner 2 the optical pin configuration was changed to optimize ineasurements of non-concentricity.

The framing camera data indicalcu that the liner remained straight as it imploded. The high-resolution gated canera inage in Figure 10, taken at $7.58 \mu$ s after current rise. shows many details including the cutout in the electrode edge and the uniformity of the liner material laid down on the electrode. The $x$-ray and optical pin data showed that the liner remained straight. cylindrical. and concentric to the initial axis within the resolution of the diagnostics. An axial radiograph as shown in Figure 11 indicates the implosion of the liner was concentric on to a diagnostic cylinder within the resolution of the diagnostic $(\sim 80 \mu \mathrm{m})$.

The timing data from the symmetry pins are shown in Figure 12 and have been translated to an equivalent height above or below the average assuming a liner velocity of $3 \mathrm{~mm} / \mu \mathrm{s}$. This data shows that any small scale irregularities of the inner surface were less than 60 um peak height. All of the Liner 2 data support the conclusion that we achieved a symmetric, concentric. unperturbed liner implosion.

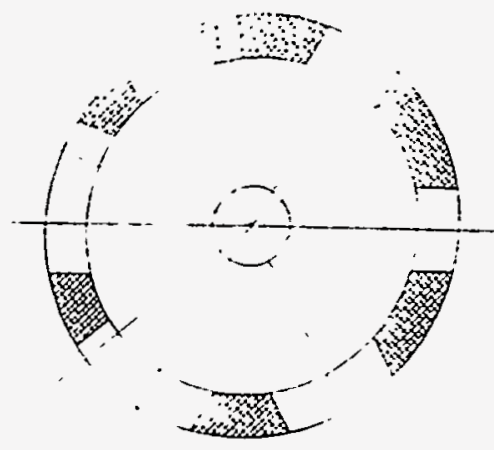

iner $O$ and Liner 1

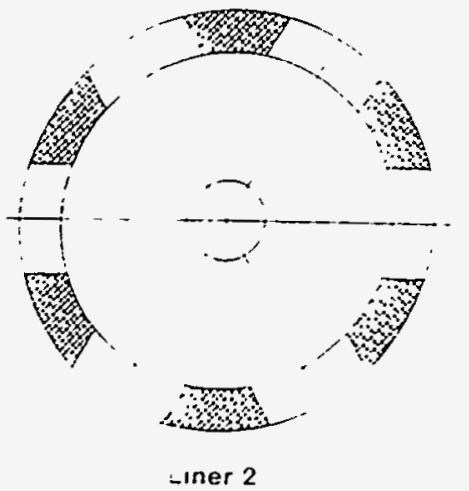

Figure 9. Diagnostic window cutout pattern in outer coaxial electrode for the different hots.

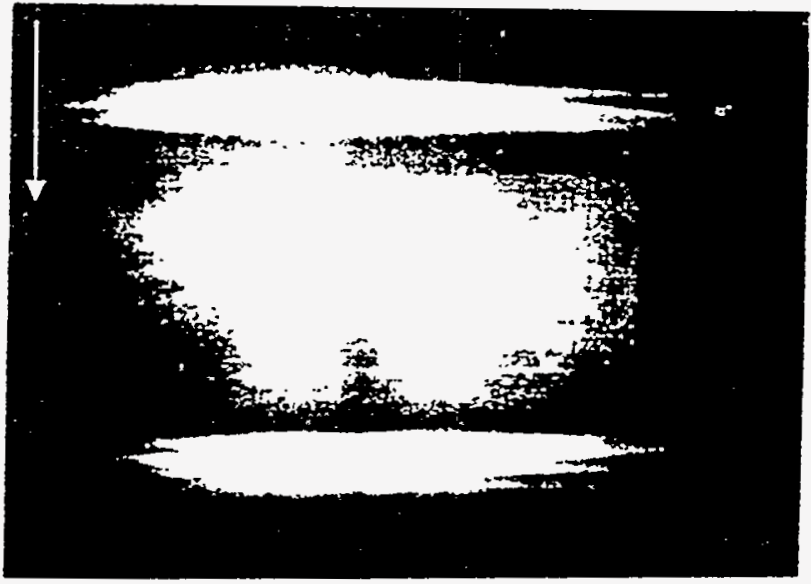

Figure 10. High resolution image of Liner 2 taken with a $\mathbf{5 0} \mathrm{ns}$ gate centered at $\mathbf{7 . 5 8} \boldsymbol{\mu s}$ after current start. The electrode notch is visible.

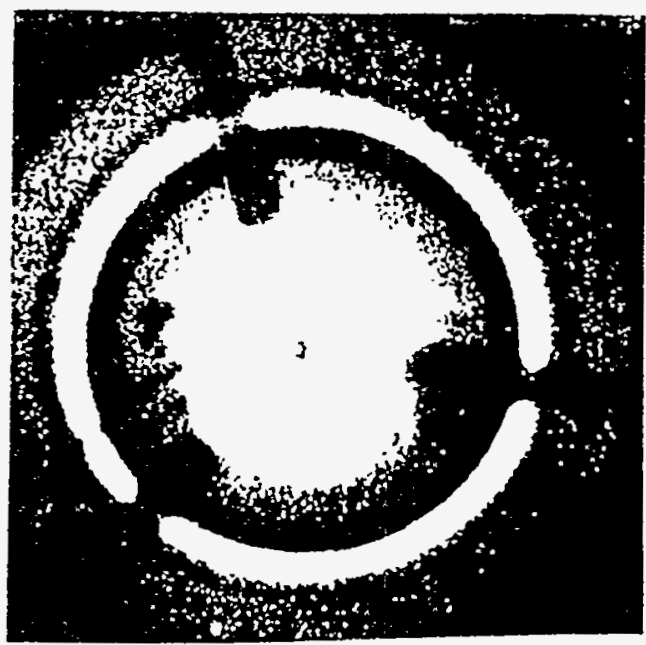

Figure 11. Axial radiograph showing liner just at the end of the elctrode gap. The pin cylinder is vivihle in the center. 


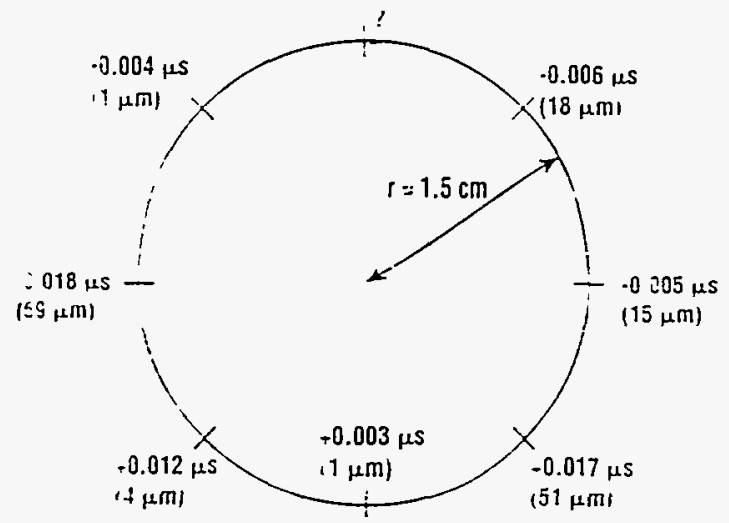

Figure 12. Timing and calculated offset of liner at $15 \mathrm{~mm}$ radius based on a velocity of $3 \mathrm{~mm} / \mu \mathrm{s}$.
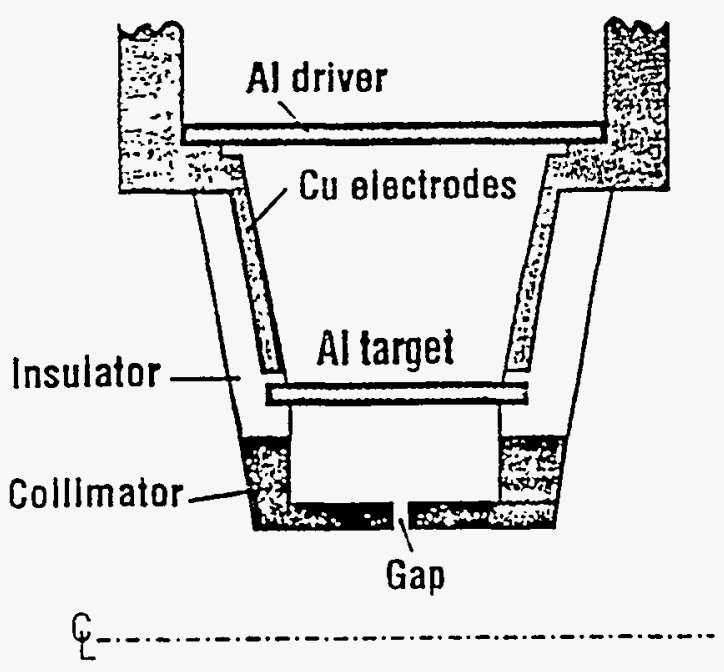

Figure 13. Load configuration for the second experimental series. The collimator, target liner, and driver liner are all concentric to the machine axis.

\section{Liner Series 2}

A second Liner series was begun at the end of January. The main purpose of the second experimental series was to characterize the target liner and develop diagnostics necessary for the shock induced quasi-particle ejecta experiments including holography. Only the results from the third shot, Pegasus II-26 fired on April 26th, from the series will be discussed. The load configuration is given in Figure 13. The concept for the Liner Ejecta experiment is that the driver liner is used to impact a target liner which then accelerates toward a collimator with a slit in it. A shock wave is set up in the target liner and as the shock emerges from the back side of the target liner, ejecta is generated. By taking a laser hologram the particle distribution of the ejecta is hoped to be determined. The goal for the second experimental series was to characterize the target dynamics and not to measure and generate ejecta.

The target liner had a 21 micro-inch surface finish on the inside. The machine was fired at $34.3 \mathrm{kV}$ with an error estimate of $+1-3 \%$. A $50 \%$ thicker fuse was used on this shot compared to the last liner series to ensure the fuse action didn't occur until after the current peak. The Rogowski current was reported as 5.3 MA based on a zero crossing of $8.3 \mu \mathrm{s}$ and the current inferred from the chicken strap was reported as 5.1 MA. The average Faraday data was reported as 4.6 MA without correction. Improved EMI shielding is needed for both the Rogowski and the Faraday diagnostics.

The radiographic film packs and their subsequent processing was modified to improve contrast for this shot. The fully interfaced and interlocked $x$-ray units were used for this shot. The radiographic data has only been analyzed by eye. The first radial radiograph was taken at $9.02 \mu \mathrm{s}$ after current start. The liner driver impacting onto the target liner is observed with the inner target radius $8 \%$ of the way in to the collimator. The data is shown in Figure 14. The second radial view, taken at $9.75 \mu \mathrm{s}$, indicates that the target liner is $59 \%$ of the way in as shown in Figure 15. The axial radiograph taken at $9.47 \mu \mathrm{s}$ shows that the target liner has moved $47 \%$ of the way in to the collimator as shown in Figure 16. The target velocity inferred from the axial and the first radial view is 4.4 $\mathrm{mm} / \mu \mathrm{s}$ and from the axial and the second radial is $3.3 \mathrm{~mm} / \mu \mathrm{s}$. The calculated velocity was $3.8 \mathrm{~mm} / \mu \mathrm{s}$. The high resolution "three eyes" camera system return good data showing nominal liner performance.

The holographic laser system had been improved from the last shot with an increase of intensity by over a factor of one hundred and a decrease in pulse width from 300 ns down to $25 \mathrm{~ns}$. An excellent hologram was taken at the shot showing the details of the calibration wires and posts but no indication of ejecta. The timing of the hologram was 140 ns earlier than planned and the target arrival was later than predicted and therefore one did not cxpect to see ejecta for this shot. 

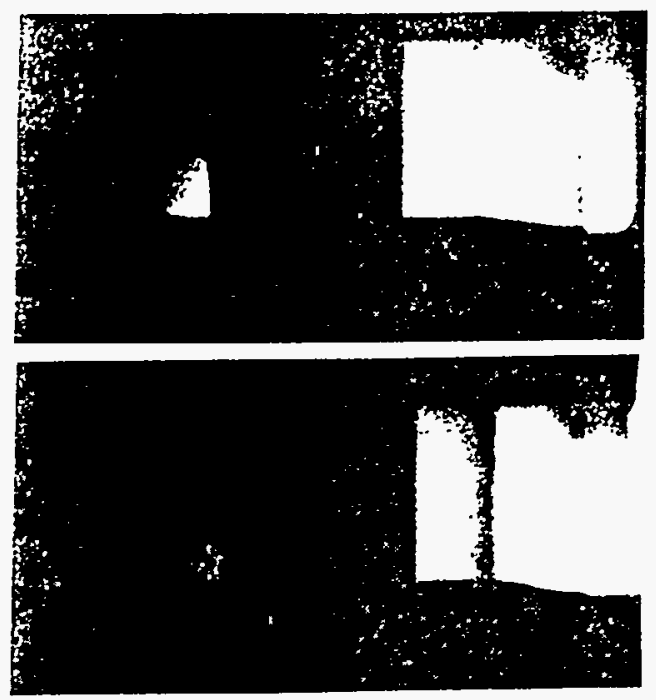

Figure 14. Radiographs taken radially before the shot and at $9.02 \mu \mathrm{s}$ after current start.
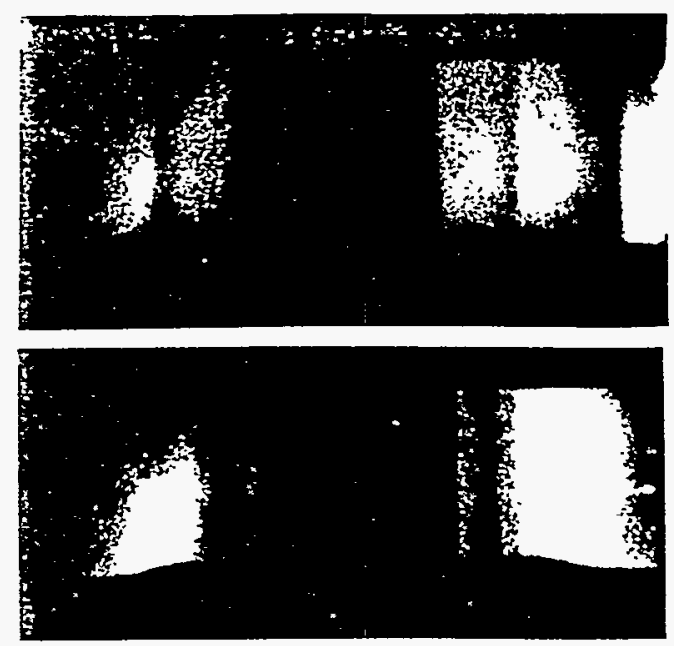

Figure 15. Radiographs taken radially before the shot and at $9.47 \mu$ s after current start.
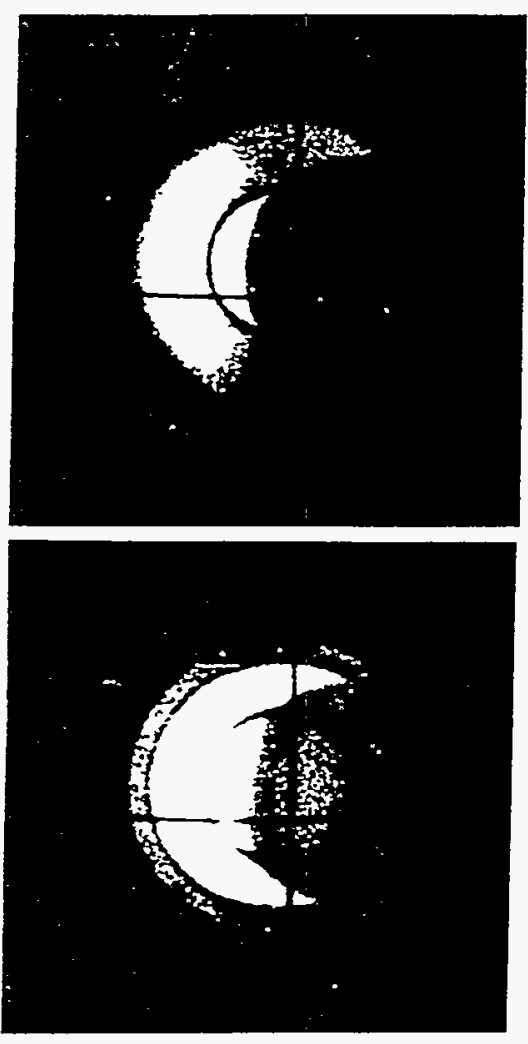

Figure 16. Radiographs taken axially before the shot and at $9.75 \mu \mathrm{s}$ after current start. 


\section{Conclusion}

We have produced and characterized a liner driver that is concentric, symmetric and unperturbed. This liner source can now be used to study hydrodynamic physics issues. The second experimental series sucessfully characterized the target dynamics necessary to move forward towards our planned quasi-ejecta experiments. We are looking forward to the building of Atlas to extend these results to higher pressures and compressable materials.

\section{Acknowledgements}

The efforts of many people from P, X, DX Divisions at Los Alamos National Laboratory and EG\&G contributed to the success of these liner series and are much appreciated.

\section{'Degnan, J. H., et al., "Multi-Megajoule Shaped Solid Liner Implosions," Megagauss Fields and Pulsed Power} Systems, pp 623, Nova Science, 1990.

${ }^{2}$ Chernychev, et al., "End Wall effects on the Shape of Imploding Cylindrical Liner," Megagauss Technology and

Pulse Power Applications, pp 707, Plenum Press, New York, 1987.

${ }^{3}$ Stokes, J., et al., "Precision current measurements on Pegasus II using Faraday Rotation," these proceedings.

${ }^{4}$ Veeser, L. , and Stokes, J. , "Fiber Optic Diagnostics for High Explosives," Proc. INWET 93 (Menlo Park, CA, November 1993).

${ }^{5}$ Platts, D., et al., "Compact Flash X-ray Units," these proceedings.

${ }^{6}$ King, N. S., et al., "Time Dependent Imaging of Cylindrical Shocks at the Pegasus Facility," these proceedings.

${ }^{7}$ Sorenson, D., et al., "In-line Particle-Field Holography at Pegasus," these proceedings. 